\title{
Upstream metadata and the impact of the IPTC schema for XMP and Adobe Bridge
}

\section{Ron Roszkiewicz}

is former president of Pound Hill Software and currently a metadata and DAM consultant in Encinitas, California.

Keywords: metadata, unstructured, IPTC, Bridge, Adobe, DAM, Creative Suite, XMP, extensible metadata platform

\begin{abstract}
Metadata discovery and modeling generally takes place at the structured, server side of digital asset management (DAM). Upstream, at the unstructured, content creation end of DAM, widespread metadata acceptance and use is still subject to the availability and understanding of schema, tools for reading and writing, and methodologies that validate the data. The inevitability of metadata becoming a key component in digital rights management, universal searches, and as a tool to leverage in the automation of tedious tasks is all but certain. A couple of important milestones along this inevitable path are represented by the recent product and technology introductions from Adobe and International Press Telecommunications Council (IPTC). An examination and analysis of the IPTC Core Schema for Extensible Metadata Platform (XMP) and the Bridge application from Adobe is the subject of this paper.
\end{abstract}

Metadata discovery and modeling usually takes place at the structured, server side of digital asset management (DAM). Upstream, at the unstructured, content creation end of DAM, widespread metadata acceptance and use is still subject to the availability and understanding of schema, tools for reading and writing, and methodologies that validate the data. It seems inevitable that metadata will become a key component in digital rights management, universal searches, and will be used as a tool to leverage in the automation of tedious tasks. A couple of important milestones along this inexorable path are represented by the recent product and technology introductions from Adobe and International Press Telecommunications Council (IPTC). The inter-related releases of the IPTC Core Schema for Extensible Metadata Platform (XMP) and Bridge application from Adobe both do their part to broaden the usability and visibility of metadata for professional and prosumers and contribute to what will inevitably be seen as a major field test of metadata.

\section{IPTC SCHEMA FOR XMP}

The IPTC schema is the result of months of workgroup activity primarily supported by Adobe Systems, IdeAlliance and coordinated under the expert management of Michael Steidl, 
Managing Director of IPTC. The fruit of their efforts is a schema that is the descendant of the original Information Interchange Model (IIM), a subset of which has been part of Adobe applications since the early 1990s. IIM itself traces its origins back to the late 1970s. The significance of this new effort is that it is a reworking of the core IIM set to become an XMP-compliant schema with properties that conform to the current needs of the image consumer and is built on the cumulative experience of users of IIM. This transformation recognizes the logic and value of XMP for the future but does not create a clean break with the past. IIM header information in legacy files is recognized by Photoshop when the file is opened and converts it to XMP when the file is saved. This is done without discarding the original IIM embedded data.

The deliverables of the IPTC/XMP workgroup is a comprehensive set of documents defining the schema and instructing in its use. There is also a set of custom File Info... panels for inputting and embedding metadata into Adobe applications. The IPTC panels can be used in other Creative Suite 2.0 (CS2) applications besides Photoshop. The entire package can be downloaded from http://www.iptc.org/IPTC4XMP/. The custom panels included with the IPTC Schema for XMP, once installed, become available in the File Info. . . area of CS2 applications (see Figure 1).

This represents a step in the right direction for IPTC and helps to validate $\mathrm{XMP}$ as a standard for the long term. What did not take place concurrent with the release of CS2 is a building out of the XMP specification. One of the main requests from users eager to adopt metadata is a way to encrypt metadata and present them as a hidden and/or read-only file to users. While it is true that devising an undefeatable security scheme for such data is probably not possible, deterrence is certainly worth the effort for most users as an interim measure. It's better than nothing and it begins the process of refinement and dialogue that has to take place if metadata are to be used for branding unstructured data with ownership and usage information. Other rights protection schemes such as watermarking are further along and seem to be getting closer to the unobtrusive, multi-layered security model metadata will have to become to be taken seriously. Since Adobe has already extended the Resource Definition Framework (RDF) on which $\mathrm{XMP}$ was based with new value types, perhaps it should make the next move in proposing a new security scheme as part of its metadata platform.

\section{BRIDGE}

Creative Suite 1.0 introduced us to lightbox-style image browsing, metadata viewing and batch processing. As a function tacked on to Photoshop it didn't receive the use and respect it deserved. In fact it was clearly a work-in-progress with its sibling Version Cue 1.0. With the introduction of Bridge, and standalone application in CS2, the situation changes completely (see Figure 2).

Bridge is a tantalizing new upstream asset management tool which for many users will serve as their first introduction to a new type of visual file management. Unlike its predecessor, the File Browser in Photoshop, Bridge is a standalone application that provides user interface customization, batch processing of color 


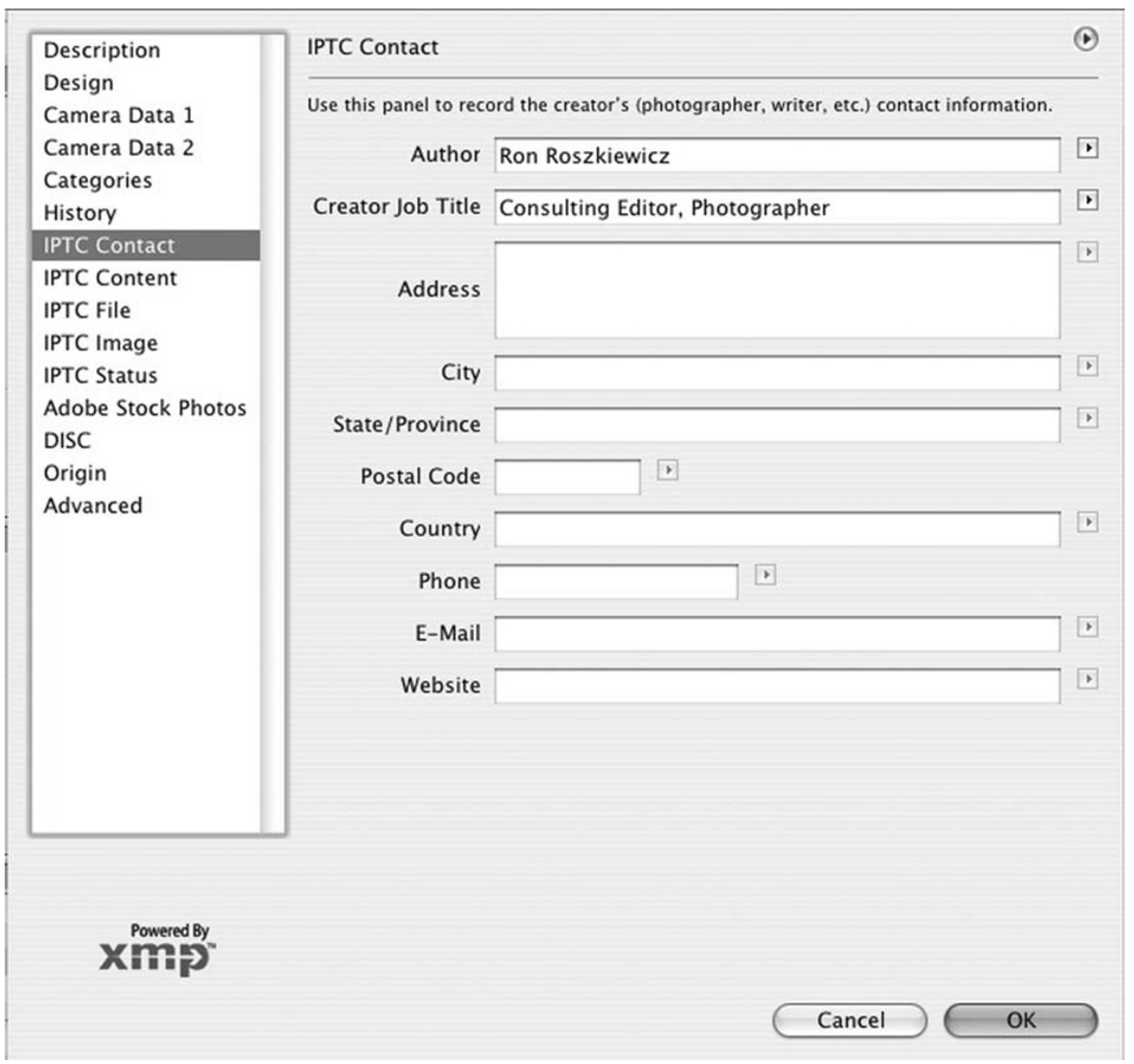

Figure 1: Contact panel from five panel set of custom panels available in File Info ... with CS2

data and metadata, saved search criteria and integration with Version Cue 2.0, CS2's versioning and file management software. With IPTC metadata panels and Bridge's File Info... function, the first widespread metadata field test is now possible. We'll find out how and why metadata are used in areas not now touched by this technology. In fact, Adobe's entry into the DAM field with this single user and workgroup solution begs the question of what will eventually constitute a low-end DAM system. Although Bridge is a refined product with no hint of the work-inprogress that Photoshop Browser exhibited, it still is something of an enigma in the sense of what its ultimate destiny will be. While it does seem, superficially, that Adobe is on a path to create a comprehensive DAM solution that rivals others in the marketplace, it's even more possible that, within the context of the CS2, they are actually building a pioneering application that will define what a low-end content 


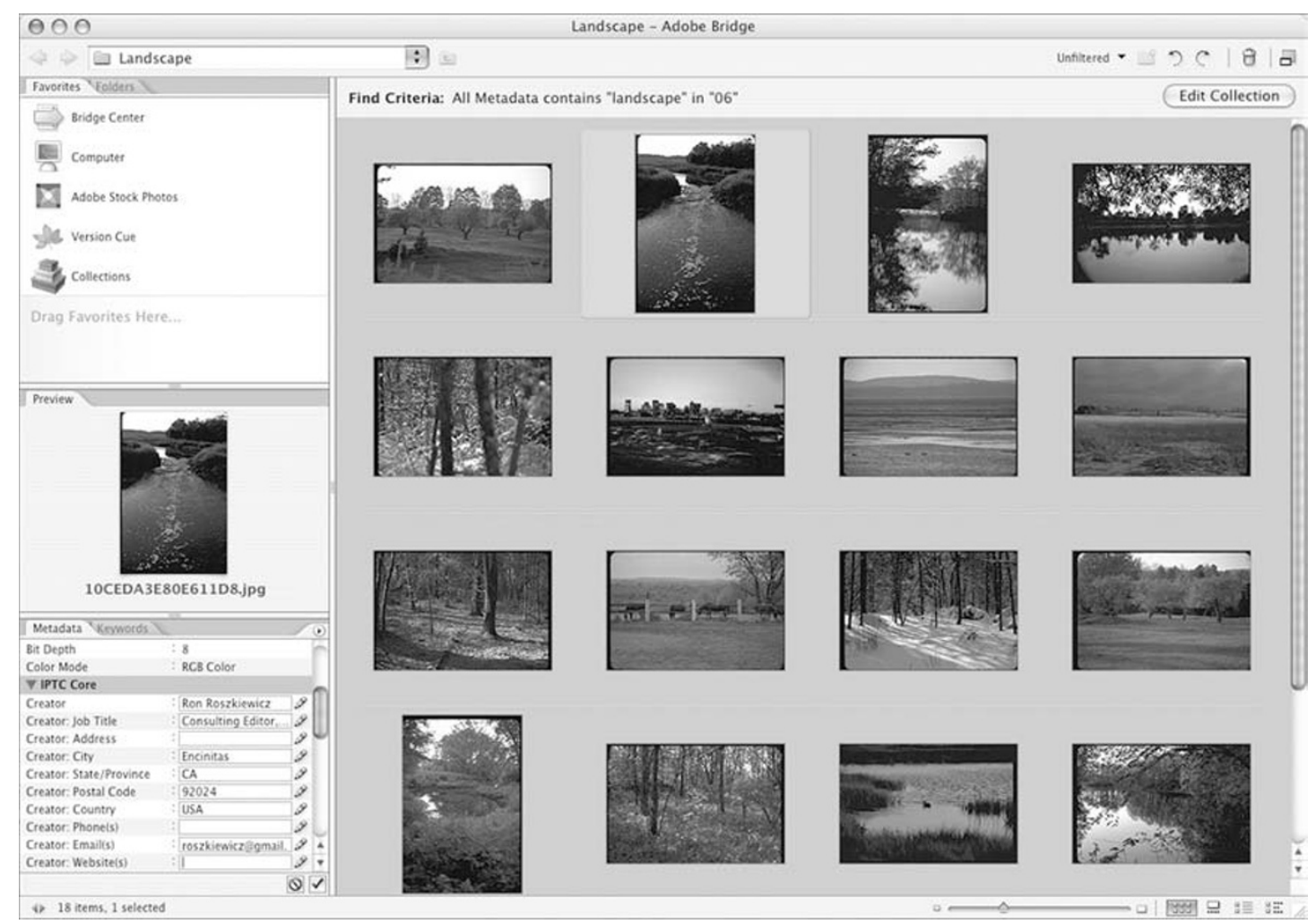

Figure 2: Adobe Bridge application with standard light box and metadata view

management system can be. When you consider that Bridge provides functionality to edit image layers, manage image versions in InDesign that are defined as alternates in Bridge, and validate content in layouts, it's clear that they could fill a void that few independent software vendors could hope to fill. Add a bit of Adobe Graphics Server technology to the mix and there are few image transformations that would not be possible. Crystal ball gazing aside, the combination of IPTC for XMP schema and Bridge provides a compelling reason for photographers to upgrade to CS2 and begin building their day-to-day workflow around it.

One of the most intriguing aspects of the Bridge package is its application programmer's interface (API). Access to
Bridge via web services is possible. This means that outside DAM systems, Bridge applications and other thirdparty applications will be able to communicate with Bridge through XML/SOAP (and other XML variants) calls. How DAM developers choose to use or ignore this new functionality will be interesting to see. It also strengthens the potential of creative IT sub-systems that link to other systems in the enterprise, eg digital rights metadata, web access, etc. Although provided as part of the CS2 suite, Bridge can also store non-Adobe file formats and video. Unlike the previous File Browser, image transformations can take place as a background process.

With Adobe's 18-month development cycle for the Creative Suite and the 
under-developed potential of Bridge in CS2, it's unlikely we will see an

enterprise DAM version of Bridge that rivals high-end DAM solutions any time in the near future. That is not to say that Bridge cannot be a legitimate partner in a multi-group, multi-node DAM implementation. Watching DAM vendors integrate Bridge into their solutions will be an interesting development to follow. The outcome of not adapting to this new reality could be the renewed emergence of data silos and webs and a general breakdown in the trend towards data centralization.

In summary, the importance of the developments mentioned above is in the activities of two important organizations to redefine the parameters of the environment for metadata use and digital asset management. Both Adobe and IPTC are mature and known for soliciting and interpreting the needs of their users through technological solutions. The combination redefines the image editing workflow and provides, through the IPTC Schema, a modern metadata infrastructure. The exciting aspect that both developments represent by the mere fact of their existence and widespread use is that we will finally have some feedback from the field to use as the basis for advancement. There are so many under-used and domain-specific schema that exist only on paper or in proprietary workflows that it will be interesting to see what happens.

We don't know yet what creative approaches will be taken to automate workflows or to overcome the real or imagined notion that creative staff will never enter metadata in a digital form. What we do know is that feedback from day-to-day use of these new technologies will provide insight that will be reflected in the next generation of Adobe products and through initiatives from industry support organizations such as IPTC and IdeAlliance (http://idealliance.org/). 